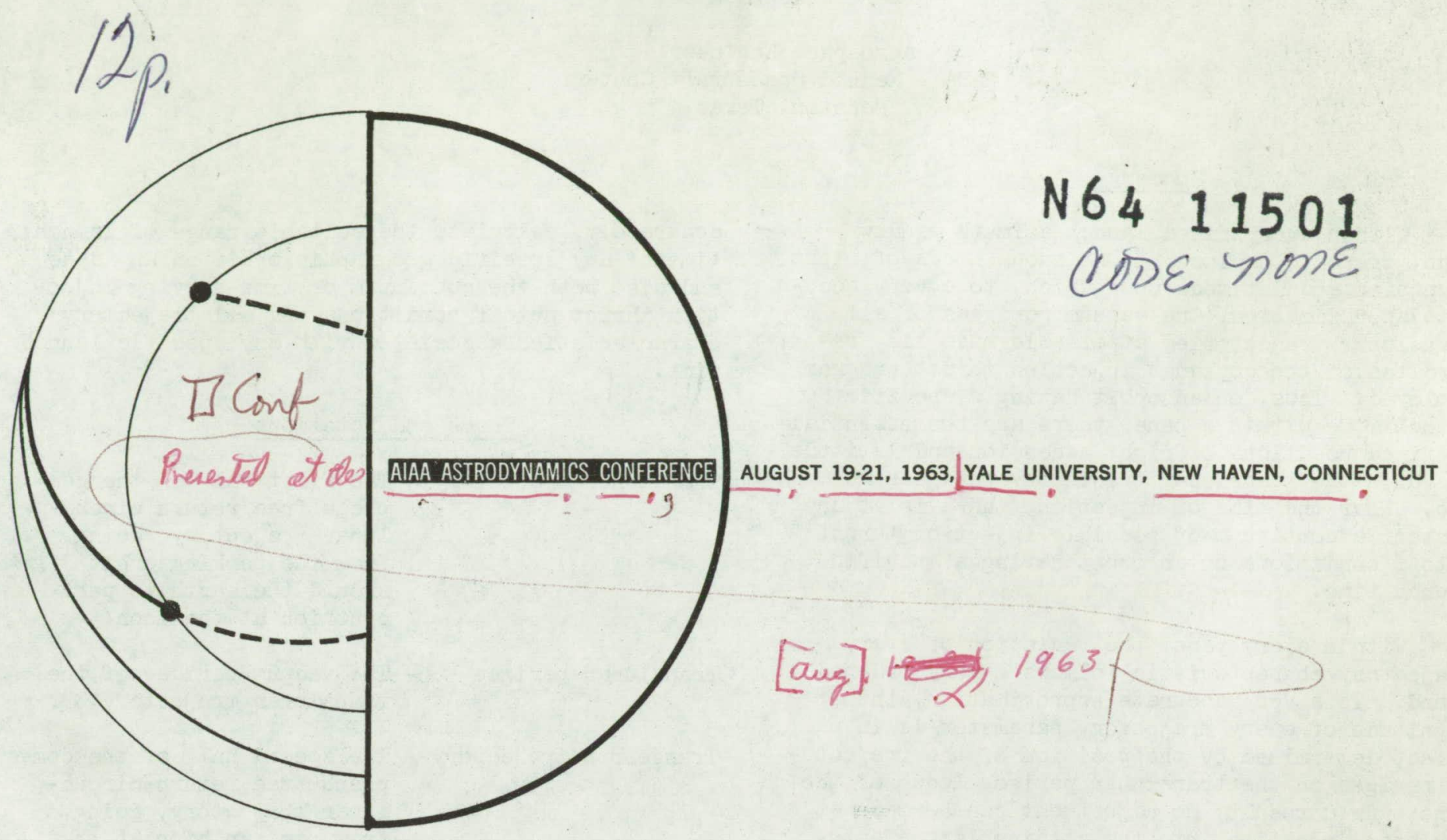

FREE RETURN CIRCUMLUNAR TRAJECTORIES FROM

LAUNCH WINDOWS WITH FIXED LAUNCH AZIMUTHS

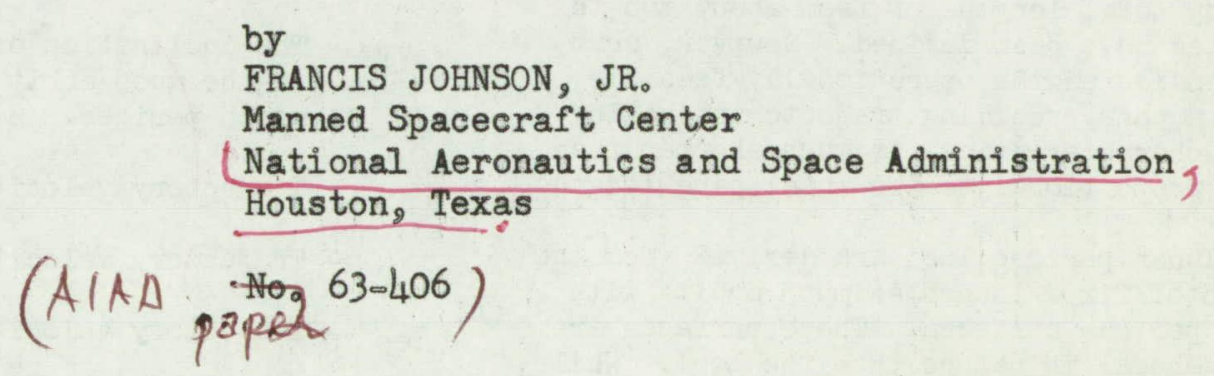


FREE RETURN CIRCUMLUNAR TRAJECTORIES FROM LAUUNCH W'

\author{
Francis Johnson Jr. \\ Aerospace Engineer \\ NASA - Manned Spacecraft Center \\ Houston, Texas
}

Summary

Within every fixed launch azimuth window pane, there is a closed, continuous locus of right ascension and latitude upon which, to a very accurate approximation, the vacuum perigees of all translunar trajectories attainable must lie, regardless of the coplanar injection thrust program employed. Thus, on an orbit having a specific launch time within a pane, there are two essentially fixed positions of right ascension and latitude at which the translunar trajectory perigees must lie. Near the time of injection, there is at any instant a continuum of possible injection thrust cutoff conditions on an orbit having a specific launch time.

Within every pane, the variation of every trajectory characteristic follows a very ordered trend. To a very accurate approximation, the magnitude of every trajectory parameter is in effect determined by the position of the trajectory perigee on the translunar perigee locus of the pane. As a result, no adjustment can be made at coplanar injection into the attainable trajectories which will change the characteristics of the trajectories; the characteristics of the attainable trajectories are in effect determined entirely by the launch time of the parking orbit within the pane. The translunar perigee locus of a pane thus presents a very comprehensive picture of all aspects of a pane, relating the variation of all trajectory characteristics to specific parking orbit launch times within the pane.

The total length of a pane is dependent upon the geometry between the equatorial plane, parking orbit plane, and the translunar perigee locus. Panes having total lengths of from about two to seven minutes have been defined. However, what would be considered the operationally feasible portion of a pane, yielding trajectories having acceptable characteristics, is much shorter than and is not proportional to the total pane length.

Translunar perigee loci are derived from the interaction of fixed launch azimuth orbits with translunar perigee surfaces. These surfaces are much more general in nature than the loci. While a translunar perigee locus represents trajectories having an essentially constant inclination to the moon orbit plane executed within a period of a few minutes on a specific launch date, a translunar perigee surface represents trajectories having an absolutely constant inclination to the moon orbit plane at any time on any date. A translunar perigee surface is the empirical representation of the characteristics of a very large group of free return circumlunar trajectories as calculated by integration. If all of the characteristics of translunar perigee surfaces were defined, it would be possible to empirically, but very rapidly and accurately, calculate the suitable range of launch time of any specific geographic orbit on any date and also both the continuum of time-varying injection thrust cutoff conditions and all trajectory characteristics associated with any specific launch time.

\section{Terms and Notations}

Translunar trajectory - The first half of the complete free return circumlunar trajectory, going from the parking orbit around the earth to pericynthion at the moon.

Translunar perigee - The vacuum perigee of the translunar trajectory.

Transearth trajectory - The second half of the complete free return circumlunar trajectory, going from pericynthion at the moon back to earth.

Transearth perigee

- The vacuum perigee of the transearth trajectory.

Pericynthion nadir - The dimensionless negative vector of the position vector of the moon at the time of trajectory pericynthion.

$i_{V T T}-$ The inclination of the parking orbit and early translunar trajectory to the moon orbit plane. See figure 2 .

$i_{V I E}$ - The inclination of the transearth trajectory to the moon orbit plane as defined at transearth perigee. See figure 2 .

$v_{i} \quad$ - Trajectory velocity at injection thrust.cutoff

$v_{l p}$ - Trajectory velocity at translunar perigee.

$v_{e p}$ - Trajectory velocity at transearth perigee.

$\gamma_{i} \quad$ Trajectory flight path angle at injection thrust cutoff.

$t_{e m}$ - Translunar flight time, from translunar perigee to pericynthion.

$t_{\text {me }}$ - Transearth flight time, from pericynthion to transearth perigee.

tee - Circumlunar flight time, from translunar perigee to transearth perigee. $\left(t_{e e}=t_{e m}+t_{m e}\right)$ 
$i_{M}$ - . The inclination of the trajectory to the monn orbit plane as defined at trajectory pericynthion. See figure 15 .

$\theta_{M}$ - The longitude of the ascending node of the trajectory plane on the moon orbit plane as defined at trajectory pericynthion. $\theta_{M}$ is measured in the moon orbit plane about the moon's center from the earth-moon line. See figure 15 .

$\phi_{M}$ - The latitude of trajectory pericynthion relative to the moon orbit plane as measured at the moon's center. See figure 15 .

$\lambda_{M}$ - The longitude of trajectory pericynthion, measured on the moon orbit plane about the moon's center from the earth-moon line. See figure 15 .

\section{Introduction}

There are two requirements of Apollo missions as presently conceived, each of which by itself imposes considerable restraints on mission planning. The one requirement is that the nominal translunar trajectory have a free coast return to earth; the nominal mission trajectory must be of the free return circumlunar type. The other requirement is that the programming of the parking orbit launch azimuth be quantized, i.e., not varied continuously, but in discrete steps throughout an overall launch azimuth window on a given launch day.

The quantity of information in both the onboard and ground-based computers associated with the programming of a specific parking orbit launch, azimuth is such that it will take several minutes for a complete cycle of storage and checkout of this information prior to launch. Due to the finite length of this storage and checkout period, it will not be possible to program a continuous variation of launch azimuth throughout an overall launch azimuth window. NLaunch azimuth programming will of necessity be quantized, taking the form of alternate GO and NO GO periods as shown in figure 1. During a NO GO period, the information is stored and checked out for a launch at a specific azimuth during the immediately following GO period. It seems only appropriate that these GO periods, being discrete elements of the overall launch window, be referred to as "window panes".

The required free return characteristic of a nominal trajectory is an obvious safety consideration. Because of the extreme accuracy requirements of injection conditions, it is not practical to expect to obtain a free return trajectory with safe reentry characteristics without some midcourse corrections. By using a free return trajectory as a nominal, it is anticipated that the required mid-course corrections will be small in magnitude and can be achieved with the attitude control jets of the Service Module. The type of free return circumlunar trajectory with which this presentation is concerned is shown in figure 2.

The objective of this presentation is to show a comprehensive picture of the factors determining the length of panes in terms of parking orbit launch time, the variation of injection conditions within different panes, and the variation of the charecter tics of all attainable free return circumlunar trajectories within different panes.

In calculating different free return circumlunar trajectories within any one pane during the course of this study, the only restrictions placed on the trajectories were that they all have the same altitudes of pericynthion and the same altitudes of transearth perigee; the trajectories were not restricted in their orientations at the moon or upon returning to earth. Injections into the translunar trajectories were always coplanar with the parking orbit. All of the trajectories involved in this study were calculated by the modified Encke method of numerical integration. It should be kept in mind throughout this presentation that these calculated free return circumlunar trajectories are highly sensitive nominals involving no midcourse corrections. The tolerances of injection velocity and times of launch and injection of these trajectories are in the order respectively of .01 to .001 feet per second and $1 / 30$ second.

\section{The Translunar Perigee Surface}

of all possible free return circumlunar trajectories of the type shown in figure 2, consider a restricted group of trajectories the members of which all have but three things in common: a specific $i_{\text {VT, }}$, a specific pericynthion altitude, and a speciflc transearth perigee altitude. No restriction is made of any other trajectory characteristic, such as an injection thrust cutoff condition, flight time, orientation at the moon or upon returning to earth, or time of performance, in defining this group of trajectories. Such a group of trajectories having these three characteristics in common are related by a very important mechanism; the vacuum translunar perigees of all trajectories within the group lie on a moving closed surface similar to that shown in figure 3 . Such a surface is referred to as a translunar perigee surface.

The inertial positions of all vacuum translunar perigees of those trajectories within the group which are executed at a specific time are represented by the position of the moving translunar perigee surface at that time. The direction of any such trajectory at its vacuum perigee on the surface is defined by the requirements that the trajectory have the $i_{V T L}$ represented by the surface and that the flight path angle be zero at perigee. The trajectory velocity at vacuum translunar perigee $\left(v_{1 p}\right)$ is in effect determined by the relative position of the perigee on the surface.

For a given set of values of $i_{V T L}$, pericynthion altitude, and transearth perigee altitude, there exists at any time a specific translunar perigee surface. The exact shape, dimensions, orientation, and motion of this surface are not only functions of the constant values of $i_{V I T}$ and the two altitudes, but also of variables such as the position and velocity vectors of the moon, the position of the sun, earth oblateness, lunar triaxiality, and even the positions of the planets: As shown in figure 4, the general shape of a translunar perigee surface in the region of low orbital altitudes is that of a very. long cone having an elliptical-shaped cross section with its apex at 
the earth's centex. The major axis of the cross section of a surface subtends about 2.7 while the minor axis subtends about $0.2^{\circ}$. The orientation and displacement of a translunar perigee surface relative to the moon orbit plane are primarily determined by the $i_{V T L}$ it represents. Due to this displacement from the moon orbit plane, the motion of a translunar perigee surface throughout a Iunar month does not lie in a plane but instead sweeps out a shallow cone, the axis of which passes through the earth's center and is perpendicular to the moon orbit plane. A translunar perigee surface rotates about this conical surface of motion throughout the lunar month roughly $140^{\circ}$ behind the moon as it moves in its orbit plane.

The extreme values of $v_{1 p}$ at a given time and altitude on a translunar perigee surface occur at the extreme edges of the surface, i.e., at the poles of the elliptical-shaped cross section of the surface at that altitude. The lowest $v_{1 p}$ at a given altitude occurs at the pole facing the general direction of surface motion, the highest $v_{\text {Ip }}$ occurring at the other pole. The $v_{1 p}$ at a given polar position on a surface (relative position between the two edges of the surface) varies at the approximate rate of 4.7 feet per second per mile of altitude change in the region of low orbital altitudes; $v_{l p}$ increases with decreasing altitude. Loci on a surface representing constant values of $v_{l p}$ take the form of angled cross sections of the surface as shown in figure 4. These iso-velocity loci are not geometrically fixed on a surface which represents any group of trajectories; they change with time as do the shape, size, orientation, and motion of the surface.

\section{Injection Conditions on A Parking Orbit Having A Specific Launch Time}

Consider a parking orbit having a specific launch time which intercepts the translunar perigee surface representing its iVT. The launch time of the orbit determines both the inertial positions of the two points of interception and their relative positions on the surface. Coplanar injections into two different free return circumlunar trajectories can be performed mathematically but not realistically from the orbit by injecting instantaneously at the times and positions of either of these two interceptions with $\gamma_{i}$ of $\infty^{\circ}$ and $v_{i}$ equal to $\mathrm{v}_{1 p}$, the value of the latter being determined by the relative position of an interception on the surface. However, in a realistic case, instantaneous injections are impossible and it is necessary to incorporate a thrust program to achieve injection into a translunar trajectory.

In the vicinity of the time of either interception of the orbit with its associated translunar perigee surface, there exists at any instant a continuum of possible injection thrust cutof $f$ conditions. This continuum of conditions is nothing more than the immediate conditions on those trajectories initiated at perigee on the translunar perigee surface at an earlier time when their perigees crossed the parking orbit plane. The only requirements of any one injection thrust cutoff condition is that extrapolating backwards in time both the motion of the vehicle on its translunar trajectory and the motion of the translunar perigee surface, the vehicle at vacuum perigee must lie on the surface and $v_{l p}$ must have the appropriate value as dictated by the relative position of the perigee on the surface. The continuum of possible injection cutoff conditions existing at any instant near the time of injection on an orbit having a specific launch time provides a great deal of freedom in injection thrust programming.

A proposed Apollo mission requirement is that for a specific orbit launch time there must be opportunities for injection on two succeeding orbits. Due to the very small difference between

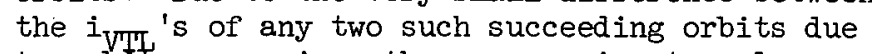
to orbit regression, the same moving translunar perigee surface can be considered as applying to both orbits. During an orbit period, the translunar perigee surface moves about $0.75^{\circ}$ while the right ascension of the ascending node of the orbit regresses about $0.4^{\circ}$ in the other direction. These opposing motions make the interceptions of the translunar perigee surface which are necessary for coplanar injection, very difficult if not impossible on both of two succeeding orbits in most cases. The most favorable conditions for interceptions on succeeding orbits would be when the translunar perigee surface is near its extreme negative or positive latitude and the $\left|i_{V T L}\right|$ involved is very small.

The Translunar Perigee Locus of A Pane Total Pane Length

In order for a specific translunar perigee surface to have application to a mission involving coplanar injection, the parking orbit must intercept the moving surface and the orbit must have the i $_{V I L}$ represented by the surface. Consider a parking orbit having a specific launch azimuth and launch time which does intercept the translunar perigee surface representing its $i_{V I L} \cdot$ By varying only the launch time of this orbit, keeping its launch azimuth and all. other orbit parameters fixed, we find that there is a range of launch time within which the orbits all intercept this surface. However, orbits having different launch times within this range do not have exactly the same $i_{V T L}$. The variation of $i_{\text {VIT }}$ within this short range of launch time is usually in the order of $0.1^{\circ}$. The small size of this variation is due to the relatively small size of the cross section of the translunar perigee surface and the fact that the surface is moving at the rate of only about $0.5^{\circ}$ per hour while the plane of the intercepting orbit moves across the surface with changing launch time at the rate of $15^{\circ}$ of right ascension per hour. A translunar perigee surface by previous definition represents a group of trajectories having one specific value of $i_{V I L}$. Thus, in the strictest sense of the word, each orbit having a different launch time within the range would be represented by a different translunar perigee surface; however, the differences between these surfaces, associated with the very small variation of ivIL, are very small and as a result these surfaces are nearly identical.

The interception of these orbits, having different launch times but the same launch azimuth and all other orbit parameters, with their nearly identical translunar perigee surfaces, defines an 
inerti.lly fixed, closed, continuous locus of right. ascension and latitude. These orbits constitute a fixed launch azimuth window pane. The inertial locus is referred to as the translunar perigee locus of the pane. An actual translunar perigee locus of a pane is shown in figure 5. A translunar perigee locus closely resembles a cross section at orbit altitude of the translunar perigee surface of any one of the orbits within the pane. However, the locus is a stretched and distorted picture of this cross section due to the slight variation of $i$ VIJ within the pane and the motions of the surface and the orbit plane. In view of the variation of $i_{V T T}$ within a pane, the $i_{V T L}$ of a pane should be referred to as a mean value.

The total length of a pane is defined as that range of launch time within which all orbits launched at the same launch azimuth will intercept their essentially identical translunar perigee surfaces (and the translunar perigee locus of the pane). The boundaries of a pane are the launch times of orbits which are tangent to their essentially identical surfaces (and the translunar perigee locus). The total length of a pane is a function of the involved geometry among the moon orbit plane, equatorial plane, and the moving parking orbit plane and translunar perigee surface(s). Total pane lengths of from two to seven minutes have been defined. The translunar perigee surfaces involved in these panes were all located very near the equatorial plane. It would be expected that longer total pane lengths would be realized in panes wherein the surfaces were near their extreme negative or positive latitudes and values of $\left|i_{\text {VIL }}\right|$ were very small. The total length of any pane would not be considered operationally feasible due to the fact that trajectory characteristics are essentially determined by orbit launch time within the pane, as is described in the following section, and within portions of every pane the characteristics of the trajectories are operationally unsuitable.

\section{Relationship Between The Translunar Perigee Locus of A Pane And Trajectory Characteristics}

Consider a parking orbit within a pane, i.e., an orbit which intercepts the translunar perigee surface representing its $i_{V T M}$. The vacuum translunar perigee of any free return circumlunar trajectory achieved from this orbit by coplanar injection must lie on this surface, the-necessary $\mathrm{v}$ lp being determined by the relative position of the perigee on the surface. If this translunar perigee surface were stationary, regardless of the injection thrust program used, the right ascension and latitude of the perigee would have to be equal to that of either of the orbit interceptions of the surface. In this hypothetical case the right ascension and latitude of these two possible perigee positions are determined entirely by orbit launch time. However, in a realistic case the translunar perigee surface is moving, but due to the relatively slow rate of surface motion (about $0.5^{\circ}$ per hour) compared to the rate of vehicle motion in the process of injecting into a translunar trajectory from the parking orbit ( $240^{\circ}$ to $340^{\circ}$ per hour), the movement of the transiunar perigee locus during the time period within which any conceivable injection process would be performed is insignificant. Consequently, to a very accurate approximation, the vacuum translunar perigees of all trajectories attainable from this orbit must lie at either of two fixed positions of right ascension and latitude regardless of the injection process used. These two possible positions correspond to the orbit intercepts of the translunar perigee surface which are determined solely by orbit launch time.

The translunar perigee locus of a pane is nothing more than the closed continuum of the right ascension and latitude of the intercepts of all possible orbits within the pane with their translunar perigee surface(s). Thus, to a very accurate approximation, the vacuum translunar perigees of all trajectories attainable within a pane, regardless of the injection process used, must lie on the translunar perigee locus of the pane; this relationship holds regardless of whether the trajectories are initiated by unrealistic but analytically convenient instantaneous injections at orbit altitude or by any realistic injection thrust program. This relationship is illustrated in figure 6 , which shows a portion of the complete translunar perigee locus shown in figure 5 . The perigees shown in figure 6 are at different altitudes, being of trajectories initiated by instantaneous injection at different $\gamma_{i}$ at orbit altitude on orbits having different launch times within the pane.

All of the characteristics of a free coast return circumlunar trajectory are strongly related to the relative position of its vacuum perigee on its translunar perigee surface. As noted earlier, the necessary $v_{1}$ of the trajectory is determined by both the polar position of the perigee on the surface and its altitude. However, all other characteristics of the trajectory are primarily determined only by the polar position of the perigee; at a given polar position, variation of the altitude of translunar perigee (and $v_{l p}$ ) has very little effect upon the other characteristics of the resulting trajectory. Consequently, all of the characteristics of all trajectories attainable within a pane are strongly related to the relative position of their perigees on the translunar perigee locus of the pane; since the position of a perigee on the locus is determined by orbit launch time, the launch time of an orbit within a pane essentially determines the characteristics of the trajectories attainable from that orbit by coplanar injection.

This relationship between the relative position of a perigee on a translunar perigee locus and several trajectory characteristics is illustrated in figure 7. Figure 7 shows the $v_{i}, t_{e m}$, and $i_{\mathrm{YTW}}$ of the same trajectories represented in figure $\frac{6}{b}$ as function of the right ascension of their translunar perigees. The general continuity of the curves in figure 7 shows that trajectory characteristics are primarily determined by perigee position on the translunar perigee locus. The ineffectiveness of the injection process in determining trajectory characteristics is illustrated by the fact that these perigees are of trajectories initiated by instantaneous injections at different positions with different $\gamma_{i}$ on orbits having different launch times. Note that the injection process has very little if any effect on $t_{e m}$ and $i_{V T E}$ while it does have a slight effect on $v_{i}$, as evidenced by the lack of overall continuity of the 
data points on the $v_{i}$ curve. The relationshifs between $t_{e m}$ and $i_{V T E}$ and translunar perigee position shown in figure 7 are typical of that between all trajectory characteristiss and perigee positions on the translunar perigee locus of any pane.

There is one trajectory characteristic, the total possible range of which is common to all panes. This characteristic is i VTF. Figure 8 shows the $360^{\circ}$ continuum of $i_{V I F}$ attainable within any pane. The relative position of a perigee on a continuous translunar perigee locus in effect determines the $i_{V T E}$ of the associated trajectory within this $360^{\circ}$ continuum. It has been found convenient to divide the translunar perigee locus of a pane into four quadrants, each representing a $90^{\circ}$ range of $i_{\mathrm{VTE}}$; trajectories having translunar perigees within any one quadrant all have values of $i_{\text {VTF }}$ within the $90^{\circ}$ range represented by that quadrant. The boundaries of these quadrants are approximated by what would be considered the major and minor axes of the elliptical-shaped translunar perigee locus. These quadrants of a translunar perigee locus are shown in figure 9. As shown in figures 8 and 9 , the terms used to identify the quadrants are the same as used to describe the orientation of the transearth trajectories relative to the moon orbit plane and the moon's orbital motion. The extreme ends of a translunar perigee locus, where the perigees of trajectories within the pane having $i_{\text {TाE }}$ of $0^{\circ}$ and $180^{\circ}$ occur, are referred to respectively as the posigrade and retrograde poles of a locus. As shown in figure 9, the extremes of other trajectory characteristics are related to these same poles.

Since a translunar perigee locus closely resembles a cross section of the transiunar perigee surface of any one of the orbits within the pane, the relationship between perigee positions on a locus and trajectory characteristics is very similar to the relationship between these same characteristics and the polar positions of perigees on the surface. A translunar perigee surface thus presents a comprehensive picture of the characteristics of a group of trajectories relative to the moon orbit plane. The surface represents the times, positions, directions, and velocities of the vacuum translunar perigees of trajectories within the group, all characteristics of these trajectories being related to the polar positions of their perigees on the surface. A translunar perigee locus on the other hand, is much more specific in that it presents a comprehensive picture of every aspect of a specific fixed launch azimuth window pane, relating all trajectory characteristics and injection conditions to the launch times of orbits on a given date.

There are several different means of representing the variation of trajectory characteristics within panes. Parameter values can be represented as double-valued functions of the right ascension of the ascending node of the parking orbit on the equatorial plane. This form of representation in effect shows the variation of the parameters as a function of orbit launch time; it is very useful in defining the useful portion of a pane based on operational restrictions of trajectory characteristics. As shown in figure 7 , it is possible to represent the variation of trajectory parameters as a function of the right ascension (or latitude) of translunar perigee. However, the translunar periggee loci of different panes differ in shape, dimension, and position. Consequently, this form of representation does not facilitate the convenient correlation of characteristics of different panes.

It has been found very convenient to represent trajectory parameters as a function of $i_{V T E}$ for several reasons. The complete $360^{\circ}$ range of $i_{V T F}$ is common to all panes." Over an absissa range of $360^{\circ}$, any trajectory parameter can be expressed as a single-valued function. Extreme values of trajectory parameters within a pane usually occur on trajectories having extreme values of $i_{V T E}$.

As shown in the latter part of this section, variations of trajectory velocity at translunar perigee and transearth perigee ( $v_{1 p}$ and $\left.v_{e p}\right)$ and the variations of translunar, transearth, ep and circumlunar flight times ( $t_{e m}, t_{m e}$, and $t_{e e}$ ) within panes, can be readily expressed as functions of $i_{\text {VTI }}$. More involved trajectory characteristics, such as orientation and geometry at the moon and transearth perigee position, while just as strongly related to $i_{\text {TrF }}$ and translunar perigee position as the forementioned velocities and flight times, are more comprehensively described as separate subjects in later sections.

As noted earlier, the total length of a pane would not be considered operationally acceptable due to the undesirable trajectory characteristics associated with portions of all panes. It has been found that what would be considered the useful portion of a pane, based on any given requirements of trajectory characteristics, is not proportional to the total length of a pane. For instance, of a pane having the total length of 126 seconds, the portion yielding trajectories having $\left|i_{V I E}\right|$ less than $20^{\circ}$ was found to be 39.6 seconds long; the similarly defined portion of another pane having a total length of 377 seconds was found to be 31.2 seconds long.

Figure 10 shows $v_{1 p}$ at an orbital altitude of 650,000 feet in different panes as a function of ivIF. As noted earlier, with variation of altitude, $v_{1 p}$ changes at the approximate rate of 4.7 feet per second per mile of altitude at a given polar position, increasing with decreasing altitude. It can be seen that the range of $v_{l p}$ at a given altitude is independent of $i_{V T M}$ but is dependent upon the position of the moon. The results indicate that $i_{\text {pr }}$ has much less effect in determining $v_{l p}$ (and $v_{i}$ ) than the position of the moon and the specific launch time of the parking orbit within the pane, the latter being represented indirectly in figure 10 by $i_{\mathrm{VTE}}$. Notice that within each pane, the range of $v_{1 p}$ of trajectories having $\left|i_{V I E}\right|$ less than $20^{\circ}$ is only about 2 feet per second as compared to the total range of $v_{1 p}$ within the pane of between 65 and 75 feet per second.

Figure 11 shows the variation of $v_{e p}$ within different panes as a function of ivTt . At a given perigee position, $v_{e p}$ has the same sensitivity to transearth perigee altitude as does Vlp to translunar perigee altitude; approximately 4.7 feet per second per mile of altitude, $v_{e p}$ increasing with decreasing altitude. There are 74 different trajectories represented in figure 11, the average absolute deviation of their transearth 
perigie altitudes from the nominal altitude of 30 ... miles being 2.21 miles. Due to the sensitivity of $v_{e p}$ to transearth perigee altitude, it was necessary to take into consideration the deviations of the individual trajectories in deriving the curves shown in figure 11. Note that within a pane, a trajectory having an $i_{V I E}$ of $\alpha^{\mu}$ will have the lowest $v_{1 p}$ and the highest $v_{e p}$, while a trajectory having an $i_{V T F}$ of $180^{\circ}$ will have the highest $v_{1 p}$ and the lowest vep.

The variations of the flight times within a typical single pane are shown in figure 12. Figure 13 shows the variation of translunar flight time within different panes. The longest translunar flight time, shortest transearth flight time, and shortest circumlunar flight time attainable within a pane occur on a trajectory having an $i_{\text {VTE }}$ of $\rho^{\rho}$. The opposite extremes of these flight times within a pane occur on a trajectory having an $i_{\mathrm{VTH}}$ of $180^{\circ}$. The size of the range of each of these three flight times within a pane is independent of $i_{V I L}$ but is dependent upon the position of the moon. For a given position of the moon, $i_{y}$ has much less effect in determining flight times than the specific launch time of the parking orbit within the pane, the latter being represented indirectly in figure 13 by $i_{\mathrm{VTW}}$. The circumlunar flight time of trajectories within a pane having $\left|i_{V T E}\right|$ less than $20^{\circ}$ is essentially a constant value; this effect can be seen in figure 12. The results shown in figure 13 indicate that a translunar flight time of about 64 hours can be achieved at any time regardless of the position of the moon; however, the $i_{\mathrm{VWE}}$ of the 64 hour trajectory reaching the moon at apogee will be approximately $180^{\circ}$.

\section{Trajectory Geometry Near The Earth Relative To The Position of The Moon At Trajectory Pericynthion}

A translunar or a transearth perigee locus by itself tells nothing about the geometric relationship between the planes and perigees of the trajectories, and the moon orbit plane and the position of the moon at the time of trajectory pericynthion. A detailed treatment of these very ordered geometric relationships within different panes is beyond the intended scope of this presentation; however, several of the more important relationships can be described briefly.

The angle between the translunar perigee and the pericynthion nadir of trajectories, in panes having mean $\left|i_{V T L}\right|$ in the order of 80 or less, is $9.76^{\circ} \pm 0.05^{\circ}$ for trajectories having $\left|i_{\mathrm{YTW}}\right|$ less than $20^{\circ}$, regardless of the position of the moon. Parking orbits which actually intercept the pericynthion nadir of their associated trajectories occur only in panes having mean|ivpl of approximately $4^{\circ}$ or less. The trajectories attainable from the parking orbit which intercepts the pericynthion nadir do not exhibit any unusual or extreme characteristics, nor do they have any unusual or extreme injection conditions. None of the parking orbits in panes having |iviL| greater than about $4^{\circ}$ intercept the pericynthion nadir.

In panes having higher values of $\left|i_{V M T}\right|$, the intersections of the orbits within the pane with the moon orbit plane tend to converge at a position approximately $1.5^{\circ}$ past the pericynthion nadir, i.e., the orbits tend to "aim" at the position of the moon two to four hours after pericynthion is actually achieved.

This same type of effect is observed in the trensearth trajectory geometry in all panes. Except in cases when $i_{\text {YTt }}$ is close to $0^{\circ}$ or $180^{\circ}$, all transearth trajectories in a pane intersect the moon orbit plane between $1^{\circ}$ and $2^{\circ}$ behind the pericynthion nadir, i.e., the transearth trajectories tend to "aim" at the position of the moon two to four hours before pericynthion was actually achieved.

\section{Variation of Transearth Perigee Position Within A Pane}

The transearth perigees of all trajectories attainable within a pane lie on a closed, continuous locus of right ascension and latitude just as do the translunar perigees. However, the shape and size of a transearth perigee locus and its orientation relative to the moon orbit plane are quite different from that of a translunar perigee locus. Figure 14 shows both the transearth and translunar perigee loci of a pane and the range of the pericynthion nadirs along the moon orbit plane; this is the same pane represented in figures 5 through 7 . The position of the transearth perigee of a trajectory on the transearth perigee locus of a pane is in effect determined by the position of its translunar perigee on the translunar perigee locus. The perigees and pericynthion nadirs of several specific trajectories within the pane are identified alphabetically in figure 14 .

The size, shape, and orientation of a transearth perigee locus have been found to be essential: ly independent of $i_{V T L}$ and the position of the moon: It is possible that the declination of the moon, and consequently the latitude of the translunar perigee surface $(s)$ and locus, have an effect on these properties of a transearth perigee locus. The general description to follow applies to panes wherein the translunar perigee surface(s) and locus are near the equator.

The shape of a transearth perigee locus is that of a distorted ellipse. What would be considered its minor axis subtends about 8.70 great circle arc and is coincident with the moon orbit plane. What would be considered its major axis subtends about $13.4^{\circ}$ great circle arc. It is geometrically symmetric about its minor axis and the moon orbit plane but not about its major axis. Its geometric center is nearly coincident with the pericynthion nadir of the trajectory within the pane having an ivTE of $180^{\circ}$.

It is not illustrated herein, but when the transearth perigee positions within a pane are normalized to their respective pericynthion nadirs, the resulting locus formed by the perigee positions is nearly a perfect circle. The center of this circular normalized transearth perigee locus lies on the moon orbit plane, but it is not coincident with either the pericynthion nadir or the intersection of the transearth trajectory planes with the moon orbit plane.

\section{Trajectory Orientation In The Vicinity of The Moon}

Within a pane, the orientation of the attainable trajectories in the vicinity of the moon 
tollows. a very ordered pattern, just as it does near translunar and transearth perigees. Parameters found convenient in descrioing the orientation of a trajectory at the moon are described in figure 15 . The variation of these parameters within a pane is strongly related to the variation of the position of trajectory perigee on the translunar perigee locus of the pane. It is possible to represent these parameters as a function of $i_{V T E}$, but this form of representation, while facilitating convenient correlation of parameter variations in different panes and indirectly relating parameter values to the translunar perigee locus, does not present a complete physical picture of trajectory orientation.

The pericynthions of all trajectories attainable within a pane lie on a continuous, closed locus of $\phi_{\mathrm{M}}$ and $\lambda_{\mathrm{M}}$. This pericynthion locus, which is not inertially fixed as are the translunar and transearth perigee loci, is nearly a perfect circle. Figure 16 shows the pericynthion locus of the pane represented in figures 14 and 5 . The size of a pericynthion locus is determined by the position of the moon, being largest when the moon is near perigee. The diameter of a pericynthion locus is between $13^{\circ}$ and $15^{\circ}$, in units of $\phi_{\mathrm{M}}$. The center of a pericynthion locus lies on the opposite side of the moon orbit plane from the early translunar trajectory; the displacement of the center in units of of $\phi_{\mathrm{M}}$ is approximately equal to 1/8 of the negative mean $i_{V T}$ of the pane. The pericynthion locus is essentially tangent to the plane which contains the earth-moon line and is perpendicular to the moon orbit plane; the locus lies on the side of this plane facing the direction of the moon's orbital motion.

The position of a translunar perigee on the translunar perigee locus of a pane in effect determines the position of the pericynthion of the same trajectory on the pericynthion, locus. Pericynthions having the extreme values of $\lambda_{M}$ on a locus are on trajectories having $i_{V T E}$ of $0^{\circ}$ and $180^{\circ}$; of these two extreme pericynthions, the one having a $\lambda_{M}$ nearest $180^{\circ}$ is on the trajectory having an $i_{V I L}$ of $\infty$. Pericynthions having extreme values of $\phi_{M}$ on a locus are on trajectories having $i_{V I E}$ of $90^{\circ}$ and $-90^{\circ}$. An interesting feature of a pericynthion locus is that the $i_{\mathrm{VTT}}$ of the associated trajectories can be read of $f$ of it with a protractor; centering the protractor at the center of the locus with its base parallel to the moon orbit plane, the position of a pericynthion as read on the protractor is within a degree or so of the $i_{\text {VIE }}$ of its trajectory.

Trajectories are far from being normal to a pericynthion locus. The trajectory azimuth at pericynthion is in the vicinity of $-90^{\circ}$. The results indicate that a trajectory azimuth of exactly $-90^{\circ}$ occurs at a pericynthion position having a $\phi_{M}$ equal to the displacement of the center of the locus from the moon orbit plane. The implication is that if the center of the pericynthion locus is displaced from the moon orbit plane, a zero value of $i_{M}$ does not occur in the pane. This in turn implies that the achievement of a zero value of $i_{M}$ necessitates a zero value of $i_{V I L}$ since the displacement of the center of the locus is approximately $1 / 8$ of the mean negative $i_{V I L}$ of the pane.
Figure 17 shows the relationship between $i_{M}$ " and $\theta_{M}$ within the same pane represented in figure 16. The general shape of the curve expressing this relationship in figure 17 is the same in all panes defined in this study. In panes having higher mean $i_{V T T} \downarrow$ the minimum value of $i_{M}$ is approximately equal to $1 / 8$ of the mean $\left|i_{V I L}\right|$ of the pane. In these same panes having higher mean $\left|i_{\text {YT. }}\right|$, the lower "corners" of the $i_{M^{-}} \theta_{M}$ curve are more rounded than those of the curve shown in figure 17. No special effort was made in this study to define the extreme peaks of an $i_{M}{ }^{-} \theta_{M}$ curve of a pane; however, the results obtained indicate several definite trends.

All of the trajectories associated with one of the two "branches" of an $i_{M}{ }^{-}{ }_{M}$ curve have transearth trajectory orientations whlch are either all above or all below the moon orbit plane (see figure 8). Extreme values of $i_{M}$ are associated with extreme values of $i_{V T E}$. The trajectory having the maximum value of $i_{M}$ on either of the two branches of an $i_{M}-\theta_{M}$ curve has an $i_{V T E}$ of either $90^{\circ}$ or $-90^{\circ}$. The two trajectories having the lowest values of $i_{M}$ on an $i_{M^{-} \theta_{M}}$ curve have $i_{V I E}$ 's of $\infty^{\circ}$ and $180^{\circ}$.

The magnitudes of the peak values of $I_{M}$ within the panes defined in this study were found to be within $0.5^{\circ}$ of $9.0^{\circ}$ in all cases. The position of the moon has a much stronger influence than ${ }^{1} \mathrm{VTL}$ in determining the magnitude of these peak $i_{M}$ values; for a given i $V$ Tr, their magnitude is greater when the moon is near perigee than when near apogee. The effect of the variation of $i_{V I T}$ for a given position of the moon upon the magnitude of the peak values of $i_{M}$ within a pane has not been completely defined. The results indicate that with increasing $\mid i_{V T T}$, the two peak values of $i_{M}$ within a pane become increasingly unequal in magnitude. This effect is related to the displacement of the center of the pericynthion locus from the moon orbit plane.

The maximum range of $\theta_{M}$ within a pane is but a few degrees more than the difference between the $\theta_{M}$ of the two $i_{M}$ peaks; this difference decreases as $\left|i_{V I T}\right|$ increases. The position of the moon has very little effect upon this difference. The range over which $\theta_{M}$ varies within a pane tends to be centered at either $46^{\circ}$ or $-132^{\circ}$; the range of $\theta_{\mathrm{M}}$ is centered about $46^{\circ}$ if the early translunar trajectories are above the moon orbit plane, and about $-132^{\circ}$ if they are below. In the pane represented in figure 17, the early translunar trajectories are above the moon orbit plane. Regardless of whether the early translunar trajectories of a pane are above or below the moon orbit plane, the branch of its $i_{M} \theta_{M}$ curve closest to a $\theta_{M}$ of $-44^{\circ}$ represents transearth trajectories below the moon orbit plane.

Considering the variation of transearth trajectory orientation, only a portion of the complete length of a pane is operationally feasible. The portion of a pane yielding trajectories having small values of $\left|i_{\text {ivTe }}\right|$ is represented by one of the two continuous lines forming the lower $1 / 2$ to $1 / 3$ of the complete ${ }^{i_{M}}{ }^{-}{ }_{M}$ curve. Such a portion associated with $\left|{ }^{\prime} \mathrm{V} I \mathrm{M}\right|$ less than $20^{\circ}$ is shown in figure 17 . 
d. The general shape of the $i_{M}-\theta_{M}$ curves, of, all panes defined in this study implies that there is a small region relative to the moon orbit plane system which is common to the planes of all trajectories at pericynthion within a pane; the characteristics of this region have not been defined. If braking into the lunar orbit occurs at pericynthion and if it is coplanar with the trajectory, all possible orbits within a pane form a belt having a small common nodal region. In a mission involving no plane change at insertion into lunar orbit, in order for the orbit to pass over a specific selenographic point, this point must lie within the operationaliy feasible portion of the orbit belt of the pane and a specific lunar orbit must be employed. The employment of a specific lunar orbit necessitates a specific position of the pericynthion of the circumlunar trajectory, which in turn necessitates a specific translunar perigee position. This in turn necessitates a specific parking orbit launch time within the pane, a very restrictive operational requirement. It would seem that some degree of plane change capability is necessary in any mission involving lunar orbits passing over a specific selenographic point.

In planning a lunar observation or landing mission involving a plane change at lunar orbit insertion, a proposed approach is to impose the range of potential plane change on the trajectory azimuth at pericynthion over the operationally feasible portion of the pericynthion locus of the pane. This defines an envelope of attainable lunar orbits within the pane relative to the moon orbit plane. Relating the selenographic system to this envelope of potential lunar orbits indicates the accessibility of selenographic points.

\section{Conclusions}

The characteristics of a free return circumlunar trajectory attained from a fixed launch azimuth window pane by coplanar injection, are to a very accurate approximation determined solely by the launch time of the parking orbit. Every trajectory characteristic is essentially a doublevalued function of orbit launch time within the pane. The relationships between orbit launch time and trajectory characteristics make it possible to use empirical models in planning missions involving fixed launch azimuth window panes and free return circumlunar trajectories.

Such an empirical model having a specific application is a translunar perigee locus. A translunar perigee locus is the continuum of all possible transearth perigee positions at orbital altitude of all such attainable trajectories within a pane. This locus can be derived by calculating about 20 or 30 integrated trajectories using instantaneous injections at $\gamma_{i}$ of $\infty$ at different "geographic" positions along the orbit (see figure 5); each such trajectory will be associated with a different orbit launch time. This empirical model presents a very comprehensive picture of total. pane length and the general relationship between all trajectory characteristics and orbit launch time within the pane. Injection conditions are only approximately represented by a translunar perigee locus since it does not depict the variation of trajectory velocity at perigee as a function of altitude nor does it define the time of an injection thrust cutoff condition. Trans- lunar perigee loci are very specific empirical models in that each one represents a specific pane; they are actually produced by the interaction of fixed launch azimuth orbits with much more general empirical models, translunar perigee surfaces.

Consider the planning of a mission involving free return circumlunar trajectories from a parking orbit, the launch azimuth and all other parameters of which are fixed except for launch time. For a specific launch time, if the position of the translunar perigee surface which represents the desired trajectory altitudes at pericynthion and transearth perigee and the $i_{V T}$ of the orbit, is known as a function of time following launch, it is possible to tell from this empirical model whether a nominal trajectory can be attained with that specific launch time. The condition necessary for coplanar injection is that the vehicle in orbit intercept this moving surface. If the relationship between trajectory velocity at perigee and translunar perigee position on the surface is known, this empirical model presents a picture of the continuum of suitable injection thrust cutoff conditions existing in any instant near the time of injection on the orbit which intercepts the surface. If the relationship between trajectory characteristics and perigee position on the translunar perigee surface are known, for this same orbit having a specific launch time, it is possible to determine from this empirical model the exact characteristics of the attainable trajectories, the general characteristics being determined primarily by orbit launch time.

The use of an empirical model of this type can greatly facilitate Apollo mission planning in that the accuracy of the calculation of trajectories by integration is achieved together with the speed of trajectory calculation by less accurate methods. The reliability of mission planning using an empirical model of this type is limited only by the accuracy of the integrated calculations from which the characteristics of the translunar perigee surface are defined and the accuracy of the empirical analytic representation of these characteristics. 

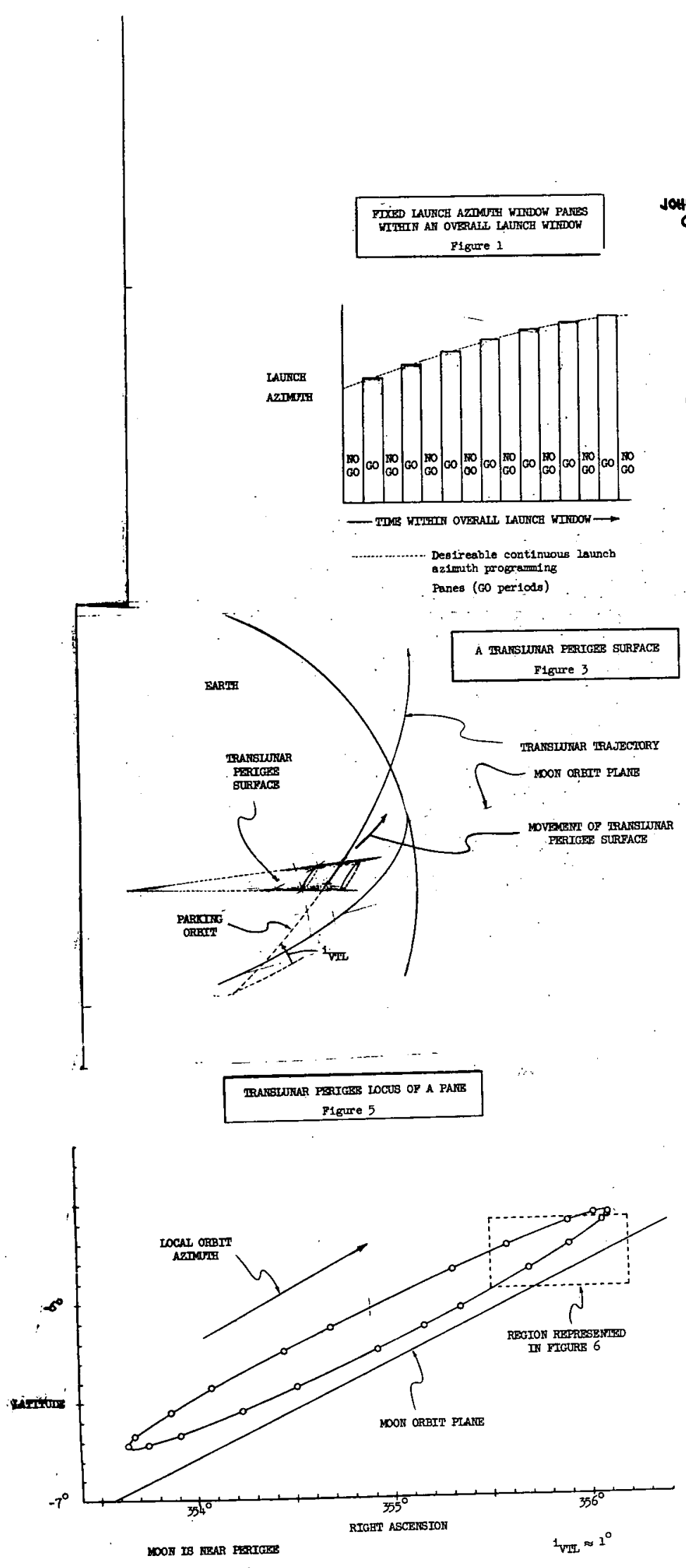

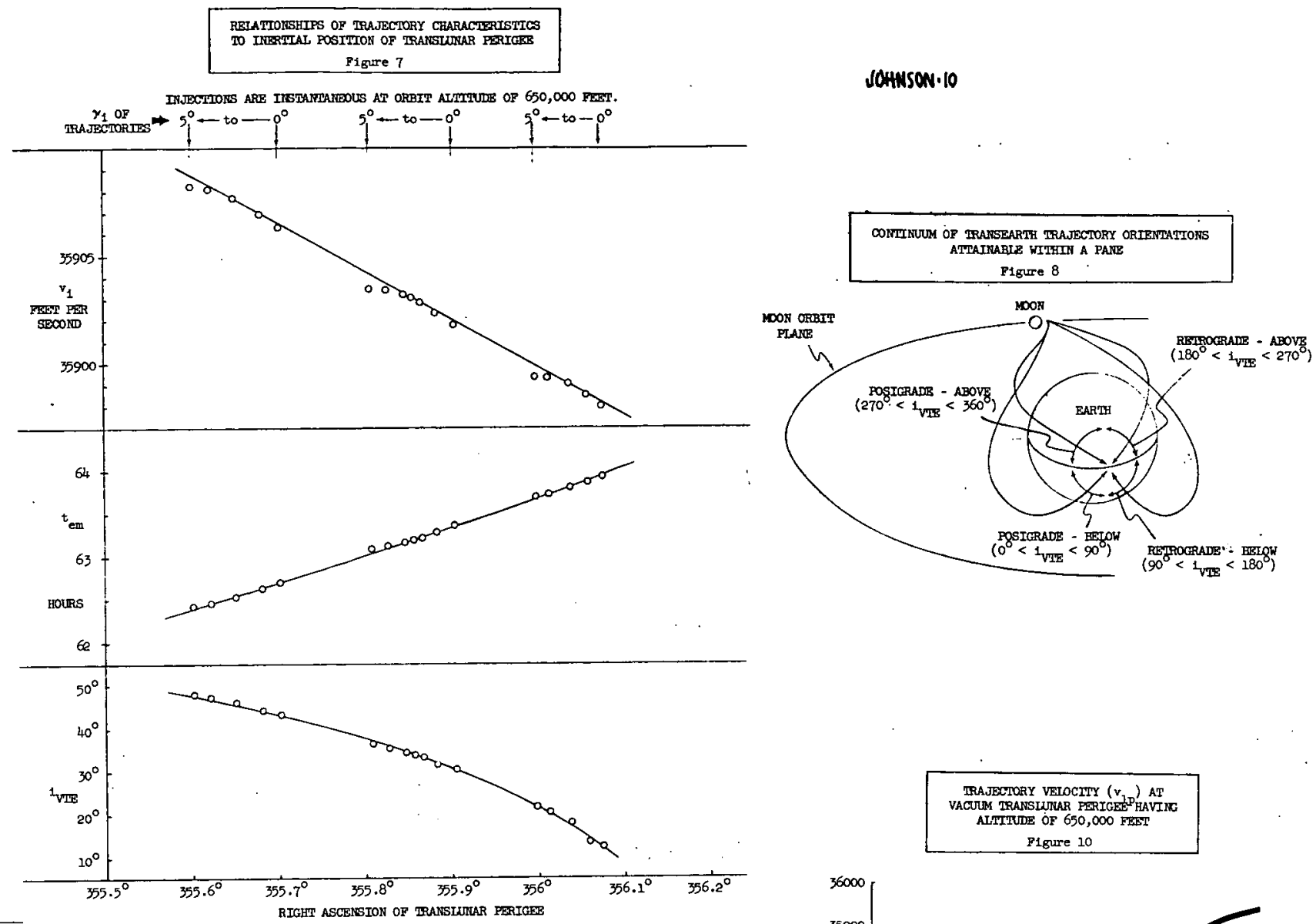

THE REIATION OF TRAJECTORY CHARACTKRISTTCS TO PERIGEE
POSITION ON A TRANSLUNAR PERIGER IOCUS Figure 9

The identification of locus quadrants and poles

denotes transearth trajectory orlentation. (Refer to figure 8)
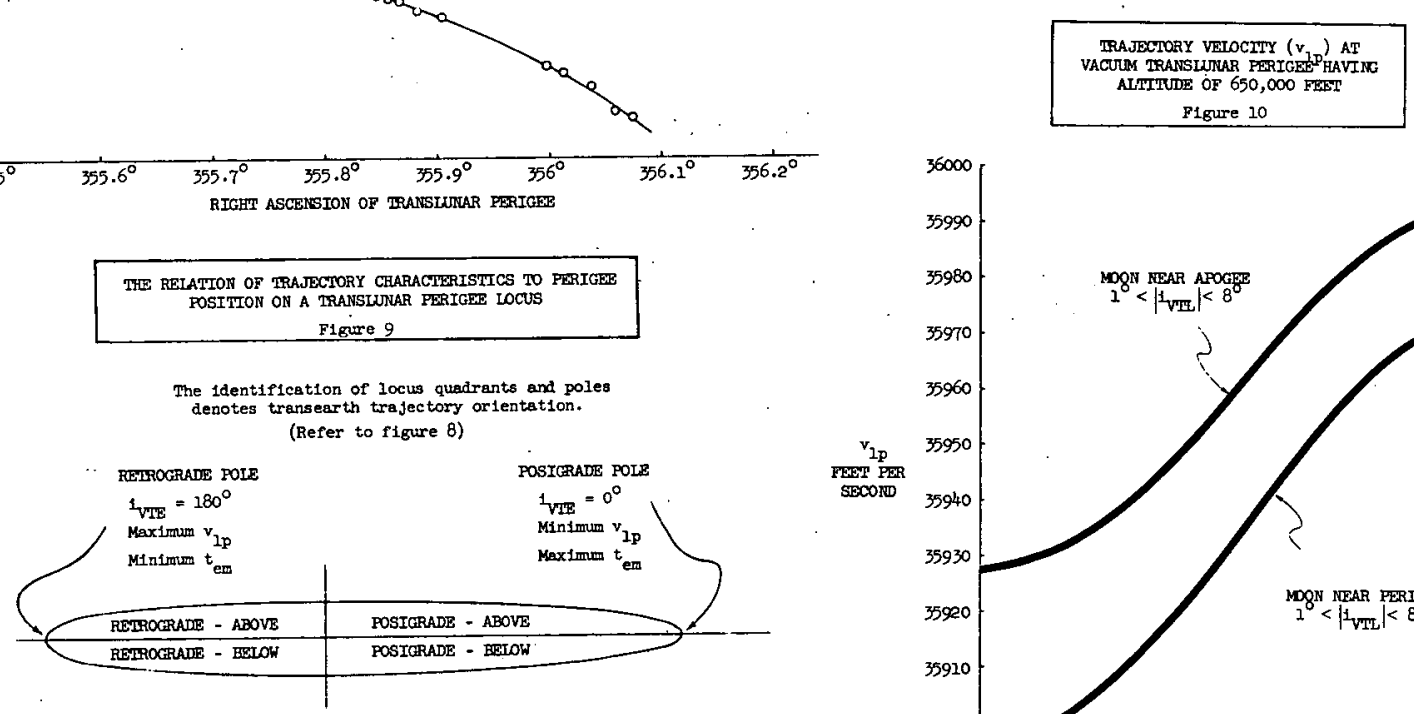

JOHWSON. 10
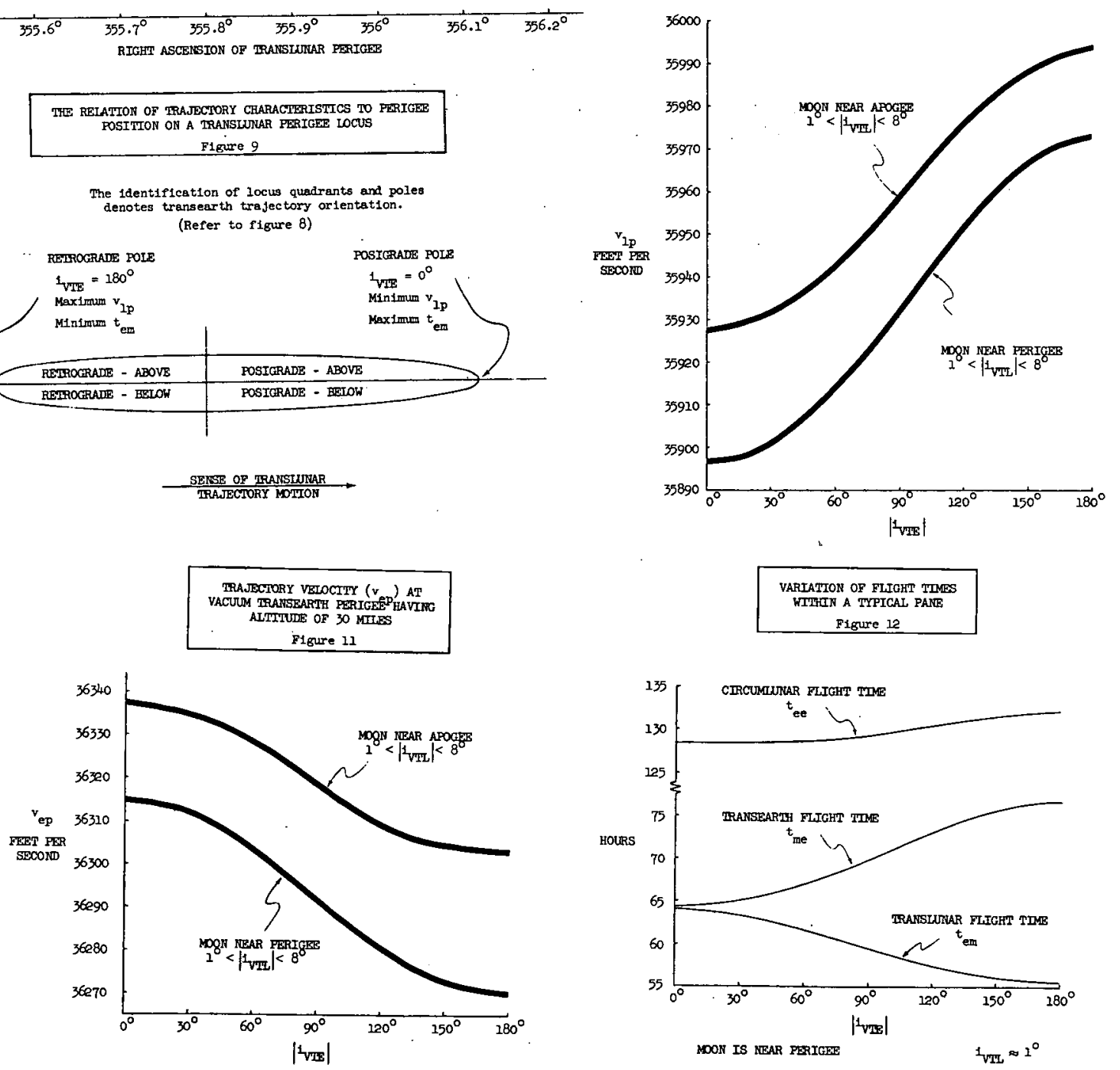


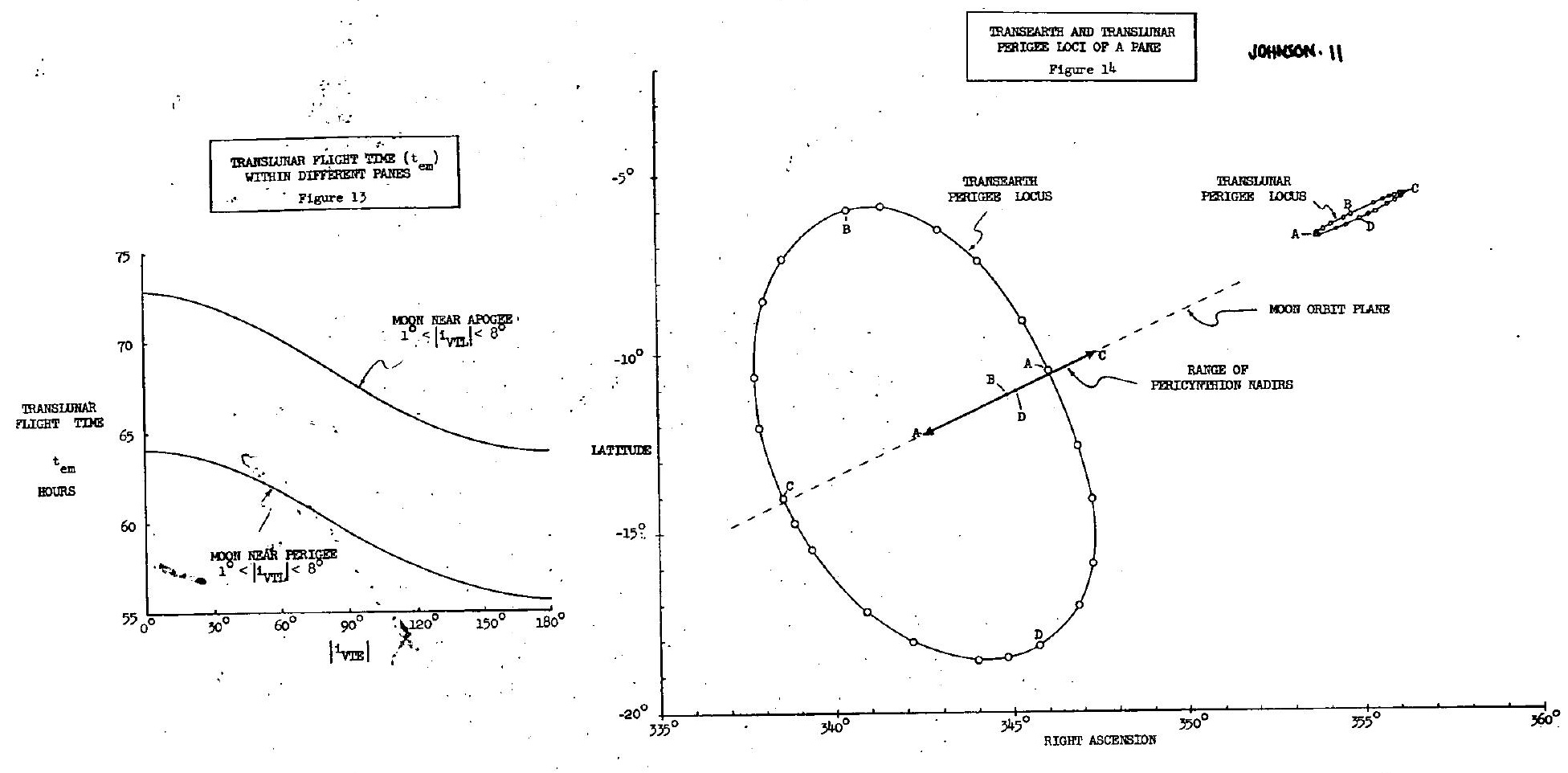

PARAMEIZRS DESCRIBING TRAJECIDRY ORIBNIATTON IN THE VICINITY OF THE MOON Figure 15

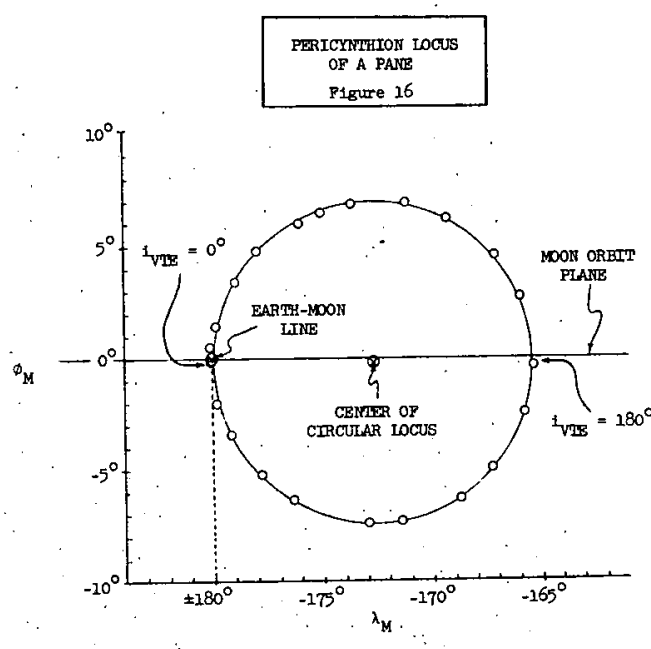

TRANSLUNAAP TRAJECTORY IS ABOVB MDON ORBIT PLANE. MDON IS ILAR PERIGER.

$1_{\text {पII }} \approx 1^{\circ}$

mox

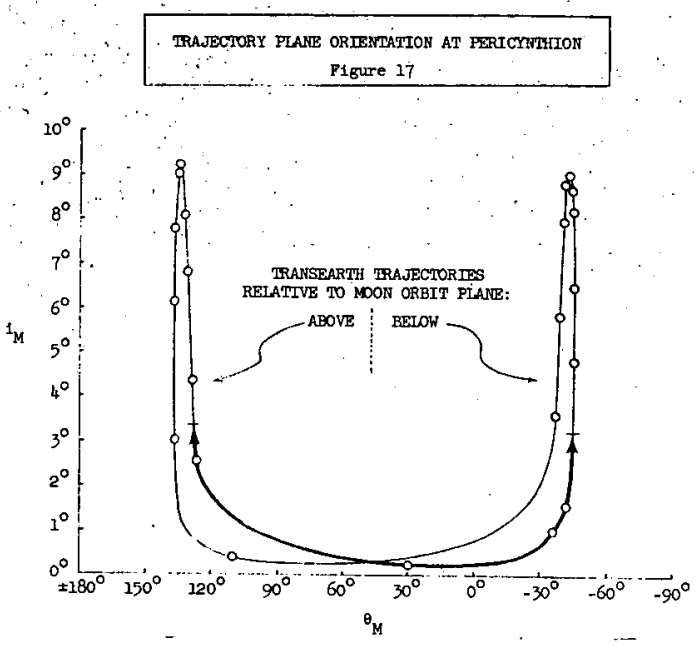

TRANSLUNAR TRAJECTORY IS ABOVE MDON ORBIT PLANE. DOON IS IRAR PERIGER.

$$
{ }^{1} \mathrm{VIT}<20^{\circ}
$$$$
{ }^{1} \cdot 1^{\circ} \approx 1^{\circ}
$$ 